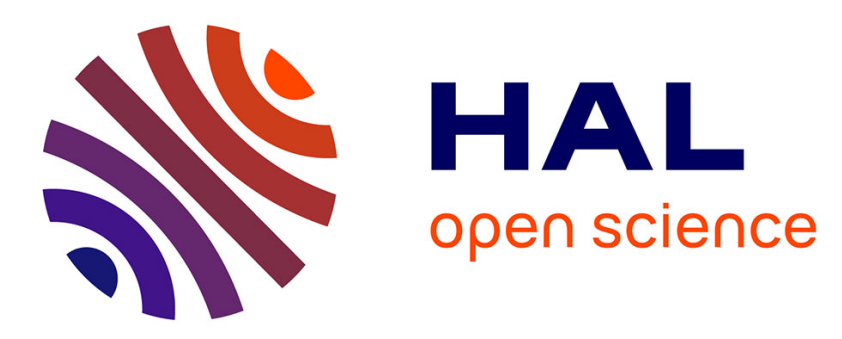

\title{
Catheter-related bloodstream infections: catheter management according to pathogen
}

\author{
Leonidia Leonidou, Charalambos A. Gogos
}

\section{To cite this version:}

Leonidia Leonidou, Charalambos A. Gogos. Catheter-related bloodstream infections: catheter management according to pathogen. International Journal of Antimicrobial Agents, 2010, 36, 10.1016/j.ijantimicag.2010.11.004 . hal-00650367

\section{HAL Id: hal-00650367 \\ https://hal.science/hal-00650367}

Submitted on 10 Dec 2011

HAL is a multi-disciplinary open access archive for the deposit and dissemination of scientific research documents, whether they are published or not. The documents may come from teaching and research institutions in France or abroad, or from public or private research centers.
L'archive ouverte pluridisciplinaire HAL, est destinée au dépôt et à la diffusion de documents scientifiques de niveau recherche, publiés ou non, émanant des établissements d'enseignement et de recherche français ou étrangers, des laboratoires publics ou privés. 


\section{Accepted Manuscript}

Title: Catheter-related bloodstream infections: catheter management according to pathogen

Authors: Leonidia Leonidou, Charalambos A. Gogos

PII: $\quad$ S0924-8579(10)00465-6

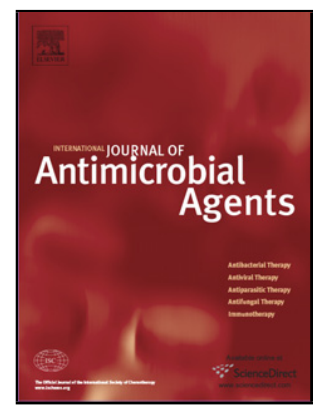

DOI: doi:10.1016/j.ijantimicag.2010.11.004

Reference: ANTAGE 3458

To appear in:

International

Journal

of

Antimicrobial

Agents

Please cite this article as: Leonidou L, Gogos CA, Catheter-related bloodstream infections: catheter management according to pathogen, International Journal of Antimicrobial Agents (2010), doi:10.1016/j.ijantimicag.2010.11.004

This is a PDF file of an unedited manuscript that has been accepted for publication. As a service to our customers we are providing this early version of the manuscript. The manuscript will undergo copyediting, typesetting, and review of the resulting proof before it is published in its final form. Please note that during the production process errors may be discovered which could affect the content, and all legal disclaimers that apply to the journal pertain. 
Catheter-related bloodstream infections: catheter management according to pathogen

Leonidia Leonidou, Charalambos A. Gogos*

Department of Infectious Diseases, Patras University Medical

School, Patras, Greece

* Corresponding author. Tel.: +326 1099 9737; fax: +3261099

9740.

E-mail address: cgogos@med.upatras.gr (C.A. Gogos). 


\section{Abstract}

Central-line access is an essential part of modern healthcare practice; however, catheter-related bloodstream infection is a major problem that causes substantial morbidity and mortality, and excess length of stay and cost. The risk of infection depends on the type of device, the site of insertion, the underlying conditions and the appropriate prevention measures taken during catheter insertion. Management of catheter-related bloodstream infection involves deciding on catheter removal, antimicrobial catheter lock solution and the type and duration of systemic antimicrobial therapy. Systemic antimicrobial use is essential but, although generally effective in controlling sepsis, it often fails to sterilize the line, increasing the incidence of complications or recurrence. The decision regarding whether the catheter should be removed or retained is therefore crucial. One of the major factors to be considered is the type of organism involved in the catheter-related infection. This review outlines the epidemiology, pathogenesis, diagnosis, microbiology and management of catheter-related infections, mainly focusing on the management of the intravascular device according to the pathogen.

Keywords:

Catheter-related infections

Catheter salvage 
Catheter management

Antibacterial agents

Page 3 of 42 


\section{Introduction}

Intravascular catheters are indispensable in current medical practice, particularly in intensive care units, but are no longer restricted to hospital inpatients. The central venous catheter (CVC) has become a lifeline for many critically ill patients, as well as chronically ill patients who have cancer or are undergoing haemodialysis.

Although such catheters provide necessary vascular access, their use puts patients at risk of local and systemic infectious complications. These devices are increasingly associated with sepsis and are the most common cause of all bloodstream infections (BSI), which have significant morbidity and mortality. Recently, the Infectious Diseases Society of America (IDSA) updated the guidelines for the diagnosis and management of intravenous catheter-related BSIs (CRBSIs).

\section{Epidemiology}

In Europe, $>60 \%$ of nosocomial infections are catheter-related, the majority of these being CVCs [1]. Since 1970 the Centers for Disease Control's (CDC) National Nosocomial Infection Surveillance System has been collecting data on the incidence and aetiology of hospital-acquired infections, including CVC-associated BSI, in a group of nearly 300 US hospitals. The data show that BSI rates are substantially higher among patients with CVCs than those without 
[2]. Rates of CVC-associated BSI vary considerably by hospital size, hospital service/unit and type of CVC.

In the USA, approximately 80000 cases of CVC-associated BSI occur in intensive care units each year, and 15 million CVC days (the total number of days of exposure to CVCs by all patients in the selected population during the selected time period) are reported in intensive care units annually. Although the consequences of these infections in terms of mortality rate remain uncertain, with a wide estimated range of $3-25 \%[2-4]$, it is clear that they increase the duration of intensive care unit stay and add a substantial burden to the cost of intensive care. The attributable cost per infection is an estimated \$34 508-56000 [5]. A total of 250000 cases of CVCassociated BSI have been estimated to occur annually if entire hospitals are assessed rather than intensive care units [6]. In this case, attributable mortality is an estimated $12-25 \%$ for each infection and the marginal cost to the healthcare system is $\$ 25000$ per episode.

\section{Types of catheter and associated mortality}

Peripheral venous catheters are the devices most frequently used for vascular access. Although the incidence of infection associated with peripheral venous catheters is usually low, serious infectious complications produce considerable annual morbidity because of the frequency with which such catheters are used [7]. However, most 
serious CRBSIs are associated with non-tunnelled CVCs percutaneously inserted into central veins, especially those placed in patients in intensive care units. The daily infection risk with CVCs is about 20 times that with peripheral catheters [8].

Short-term catheters ( $<14$ days) are the most commonly used CVCs and account for most CRBSIs. Long-term CVCs that are surgically implanted (e.g. Hickman, Broviac or Groshong catheters) appear to have one-quarter the daily risk associated with percutaneous CVCs, but still pose a much higher risk than peripheral catheters.

Peripherally inserted central catheters seem to have a similar risk of infection to CVCs in intensive care units, whereas midline catheters are associated with lower rates of infection. A lower rate of infection is also detected with totally implantable devices (ports or reservoirs) compared with CVCs. Peripheral arterial catheters, commonly used to determine blood gas levels in critically ill patients and in pressure monitoring systems, are both associated with epidemic and endemic nosocomial BSIs. Pulmonary artery catheters appear to have rates of BSI similar to those with CVCs. The highest rates of infection are reported for short-term non-cuffed and nontunnelled haemodialysis catheters $(8.0 \%, 4.8$ per 1000 devicedays), intra-aortic balloon pumps (3.0\%, 7.3 per 1000 device-days) and left ventricular assist devices (21.6\%, 2.1 per 1000 devicedays) [9]. 
Apart from the type of intravascular device, factors that partially account for the marked differences in daily infection risk rates are the experience and education of the staff, the duration of catheter placement and the clinical state of the patient [10]. Total parenteral nutrition, triple-lumen catheters and certain catheter insertion sites (jugular and femoral) seem to be independent risk factors [11].

Other factors are prolonged hospitalization before catheterization, heavy microbial colonization at the insertion site or the catheter hub, and the presence of neutropenia.

\section{Microbiology}

The microbes that colonize catheter hubs and the skin surrounding the insertion site are the source of most CRBSIs [6-8]. Migration of skin organisms from the insertion site into the cutaneous catheter tract, with colonization of the catheter tip, is the most common route of infection for peripherally inserted short-term catheters $[21,22]$. Contamination of the catheter hub contributes substantially to intraluminal colonization of long-term catheters $[12,13]$. Other routes of infection are haematogenous seeding from a distant infection or, rarely, contaminated infusate. Approximately $65 \%$ of catheter-related infections originate from the skin flora, 30\% from the contaminated hub and $5 \%$ through other pathways [14].

Scanning and transmission electron microscopy have shown that virtually all indwelling CVCs are colonized by microorganisms 
embedded in a biofilm matrix [15]. The organisms most commonly isolated from catheter biofilms are Staphylococcus epidermidis, Staphylococcus aureus, Candida albicans, Pseudomonas aeruginosa, Klebsiella pneumoniae and Enterococcus faecalis [16].

The nature of the fluid administered through CVCs may affect microbial growth. Gram-positive organisms (S. epidermidis, S. aureus) did not grow well in intravenous fluids, whereas the Gramnegative aquatic organisms (e.g. P. aeruginosa, Klebsiella spp., Enterobacter spp., Serratia spp. and Pantoea spp.) sustained growth [16]. The number of organisms on the catheter tip is related to the occurrence of BSI, supporting the concept of a critical level for biofilm development, above which substantial cell detachment and embolism occur [16].

The early stages of biofilm formation in devices involve the adherence of bacteria to cellular or matrix surfaces [17]. Further biofilm organization into complex structures is regulated by the exchange of chemical signals between bacterial cells in a process known as quorum sensing $[18,19]$. The multicellular bacterial arrangement is associated with increased resistance to antibiotics. A critical pathogenic step is the adherence of S. aureus to host cells or polymer surfaces and the subsequent accumulation of toxinproducing bacteria that promote attachment to host proteins such as laminin and fibronectin. Coagulase-negative staphylococci adhere to polymer surfaces more readily than other pathogens and certain 
strains produce an extracellular polysaccharide referred to as 'slime', which acts as a barrier against polymorphonuclear cells and binds antimicrobial agents, preventing their contact with the organism cell wall [20].

Candida albicans remains the fungal species most commonly associated with biofilm formation $[21,22]$, and the increase in Candida infections in recent decades has almost paralleled the increase and widespread use of a broad range of implanted medical devices. Different strains of C. albicans and different Candida spp. differ in their capacity to form biofilms [23]. Mature Candida biofilms exhibit a complex three-dimensional structure and display extensive spatial heterogeneity $[21,23,24]$. The overall architecture of the biofilm may vary depending on the substrate on which it is formed and its growth conditions [25]. Candida biofilms have been shown to be resistant to the action of clinically important antifungal agents, including amphotericin B and fluconazole [26].

P. aeruginosa is considered to be a model organism for the study of the diverse bacterial and molecular mechanisms that cause the switch from planktonic growth to a biofilm phenotype and contribute to bacterial persistence. Recent studies show interactions between C. albicans and P. aeruginosa in which P. aeruginosa forms a dense biofilm on C. albicans filaments and kills the fungus, but neither binds to nor kills yeast forms of C. albicans [27]. Furthermore, a P. aeruginosa quorum-sensing molecule ( $\mathrm{N}$-(3-oxododecanoyl) 
homoserine lactone) and structurally related compounds have been shown to be capable of suppressing filamentation in C. albicans cells [28].

A recent European study found that the microorganisms most often isolated from intravascular catheters are coagulase-negative staphylococci (30-51.5\%), followed by Candida spp., S. aureus, Enterococcus spp. and Pseudomonas spp. [14,16,29]. Acinetobacter spp. [30], Enterobacter spp., K. pneumoniae and other bacteria have been identified less frequently [14]. Another recent European study of intravascular catheter-related infections found that fungi (mainly C. albicans) are the third leading cause of catheter-related infections, representing the second highest colonization-to-infection rate and the highest overall crude mortality [31]. Gram-negative bacilli accounted for $19 \%$ of catheter-associated BSIs during 19861989 [32], compared with 14\% during 1992-1999 [33]. An increasing percentage of intensive care unit-related isolates are extended-spectrum $\beta$-lactamase-producing Enterobacteriaceae, particularly Klebsiella pneumoniae [34].

A recent report describing the frequency of select antimicrobial resistance patterns among pathogens reported to the CDC National Healthcare Safety Network from January 2006 to October 2007 gave the rate of occurrence among device-related infections of the following resistant isolates: meticillin-resistant S. aureus (MRSA; $56 \%$ of S. aureus); vancomycin-resistant enterococci ( $80 \%$ of E. 
faecium, $7 \%$ of E. faecalis); P. aeruginosa resistant to ceftazidime and quinolones, or piperacillin, or carbapenems $(30 \%, 17 \%$ and $25 \%$, respectively). In the same report the rates of carbapenemresistant A. baumannii and K. pneumoniae were $29 \%$ and $10 \%$, respectively [35].

\section{Diagnosis}

The diagnosis of CVC-associated infection traditionally relies on clinical features, quantitative cultivation, Gram staining and acridine-orange leucocyte cytospin testing of blood samples [3-5], or on methods that can only be applied following CVC removal $[14,36,37]$. The international reference diagnostic method is Maki's semi-quantitative method, which is based on removing the catheter and rolling the distal tip back and forth on an agar plate [38]. The growth of 14 colony-forming units (CFU) defines an insertion site infection. Growth of $10^{5} \mathrm{CFU}$ from a $5 \mathrm{~cm}$ segment of the catheter tip by semi-quantitative (roll-plate) culture, or growth of $10^{2} \mathrm{CFU}$ from a catheter by quantitative (sonication) broth culture, reflects catheter colonization. Definitive diagnosis of CRBSI requires that the same organism grows from at least one percutaneous blood culture and from a culture of the catheter tip.

The CDC recommends one of two blood culture techniques for diagnosing CRBSI when retention of the catheter is desirable paired quantitative blood cultures or paired qualitative blood 
cultures observing a differential time to positivity. For quantitative blood cultures, CRBSI is best defined by a colony count of microbes grown from blood obtained through the catheter hub that is at least threefold greater than the colony count from blood obtained from a peripheral vein [39]. Using differential time to positivity, CRBSI is best defined by growth of microbes from a blood sample drawn from a catheter hub at least $2 \mathrm{~h}$ before microbial growth in a blood sample obtained from a peripheral vein. If a blood sample cannot be drawn from a peripheral vein it is recommended that two blood samples should be drawn through different catheter lumens. Alternatively, two quantitative blood cultures of samples obtained through two catheter lumens in which the colony count for the blood sample drawn through the first lumen is at least threefold greater than that obtained from the second lumen should be considered to indicate possible CRBSI [39].

\section{Management}

The management of CRBSI involves making decisions about retaining or removing the catheter and the type and duration of the antimicrobial therapy. Determining whether the catheter should be removed or retained in place with antibiotic lock therapy (see below) in patients with CRBSI should take at least three factors into consideration: the type of CVC (whether it is short-term, arterial, long-term or port), the status of the host and the type and microbiology of the infection. 
Peripheral intravenous catheters with associated pain, induration, erythema, or exudate should be removed [39]. For a patient suspected of having short-term CVC infection or arterial catheter infection the catheter should be removed if the patient has unexplained sepsis or erythema overlying the catheter insertion site, or purulence at the catheter insertion site, or the CRBSI is associated with either suppurative thrombophlebitis, endocarditis or osteomyelitis.

Long-term central catheters or ports should be removed from patients with CRBSI associated with any of the following conditions: severe sepsis; suppurative thrombophlebitis; endocarditis; bloodstream infection that continues despite $72 \mathrm{~h}$ of antimicrobial therapy to which the infecting microbes are susceptible; or if a port abscess is diagnosed [39]. In uncomplicated cases, given the limited access in many patients who require long-term intravascular access for survival (e.g. haemodialysis), salvage of the catheter can be attempted. Both systemic and antimicrobial lock therapy should be used, repeated blood cultures obtained and the catheter removed if blood cultures remain positive for a microorganism when drawn $72 \mathrm{~h}$ after initiation of appropriate therapy.

Antibiotic lock therapy for CRBSIs is often used in conjunction with systemic antibiotic therapy and involves instilling into the catheter lumen a high concentration of an antibiotic to which the causative pathogen is susceptible [14]. Antibiotic solutions that contain the 
desired antimicrobial agent in a concentration of $1-5 \mathrm{mg} / \mathrm{mL}$ are usually mixed with $50-100 \mathrm{U}$ of heparin in sufficient volume to fill the catheter lumen (usually 2-5 mL) and are instilled or 'locked' into the catheter lumen during periods when it is not being used (e.g. for a $12 \mathrm{~h}$ period each night). Several open trials of antibiotic lock therapy for tunnelled catheter-related bacteraemia, with or without concomitant parenteral therapy, have reported response and catheter salvage without relapse in $138(82.6 \%)$ of 167 episodes $[14,40,41]$.

While awaiting blood culture results, empirical therapy to cover staphylococci and Gram-negative bacilli is the best initial treatment. In addition, for suspected CRBSI involving femoral catheters in critically ill patients, empirical therapy should include coverage for Candida spp. [39]. The regimen may be modified once the pathogen is identified. The type of pathogen causing the CRBSI is an important factor in the choice of the type and duration of therapy. Decisions as to whether the CVC should be removed or retained, the use of antibiotic lock therapy and the duration and type of therapy depend on the type of organism causing the CRBSI.

\section{Specific organisms}

\subsection{Coagulase-negative Staphylococcus spp.}

Coagulase-negative staphylococci (e.g. S. epidermidis) are the most common pathogens in CRBSIs, possibly because they appear to 
have the best adherence to inert surfaces. If a catheterized patient has a single positive blood culture that grows a coagulase-negative Staphylococcus sp., then additional cultures obtained through the suspected catheter and from a peripheral vein should be performed to be certain that the patient has true BSI and that the catheter is the likely source [42]. To ascertain the diagnosis of bacteraemia, at least two positive blood cultures, including one drawn from a peripheral vein, are necessary.

As coagulase-negative staphylococci are organisms of low virulence, removal of the line may be sufficient to resolve the infection but the patient should usually be treated with 1 week of intravenous antibiotics. Alternatively, patients with uncomplicated CRBSI can be observed without antibiotics if they have no intravascular or orthopaedic prostheses, the catheter is removed and additional blood cultures are obtained after catheter withdrawal to confirm the absence of bacteraemia [39]. According to IDSA guidelines, if the CVC is to be retained, a longer duration of therapy consisting of 1014 days rather than 5-7 days if the CVC is removed is to be considered with antibiotic lock therapy. This may control the infection in $80 \%$ of cases; however, if bacteraemia or fever persists despite appropriate antimicrobial therapy, the catheter must be removed [42]. In some cases of outpatient management of CRBSI with coagulase-negative staphylococci, when blood cultures have become negative and signs of sepsis have resolved, an oral 
antibiotic (e.g. a fluoroquinolone or linezolid) and an antibiotic lock left in place for 24-48 h have been successfully used [43].

Vancomycin is the most frequently used antistaphylococcal antibiotic for systemic and local catheter lock therapy in healthcare settings with an increased prevalence of MRSA; for institutions with a preponderance of MRSA isolates that have vancomycin minimum inhibitory concentration values $>1 \mu \mathrm{g} / \mathrm{mL}$, alternative agents such as daptomycin should be used [39]. An open-label, randomized trial that compared linezolid to vancomycin, oxacillin or dicloxacillin in the treatment of seriously ill patients with CRBSIs showed that patients treated with linezolid had a higher chance of death than patients treated with any comparator antibiotic [44]. The difference was found to be due to the inferiority of linezolid in the treatment of mixed Gram-negative/Gram-positive infections. In participants whose infection was caused by Gram-positive bacteria alone, linezolid was as safe and effective as vancomycin [45]. In light of these results the US Food and Drug Administration issued an alert for linezolid, which has not been recommended for empirical therapy in the recent guidelines.

Catheter-related bloodstream infection due to Staphylococcus lugdunensis, an uncommon cause of catheter-related infection, can cause endocarditis and metastatic infections similar to those caused by S. aureus [46] and are managed accordingly. 


\subsection{Staphylococcus aureus}

An increased risk for S. aureus infection of CVC has been described for several patient groups. Patients with end-stage renal disease requiring haemodialysis or peritoneal dialysis are at higher risk of S. aureus catheter infections, especially those who are nasal carriers [47].

If a non-tunnelled CVC is suspected to be the source of S. aureus bacteraemia, the device should be removed and a new catheter inserted at a different site. Similarly, tunnelled CVCs should be removed if there is evidence of tunnel or exit-site infection. Patients with uncomplicated CRBSIs or septicaemia caused by S. aureus should be treated for a minimum of 10-14 days after catheter removal [39]. The strongest predictor of complicated S aureus bacteraemia is the persistence of fever and/or bacteraemia for more than $72 \mathrm{~h}$ after catheter removal and initiation of antibiotics [48$50]$.

In a subset of patients with a surgically implantable catheter and no other available vascular access, or with profound thrombocytopenia, consideration should be given to catheter salvage and antibiotic lock therapy for at least 14 days [48]. Catheter guidewire exchange should be done if possible. A combination of antibiotic lock therapy and systemic therapy has been used to salvage infected ports and long-term catheters for some patients with S. aureus CRBSI 
$[40,51,52]$. However, in a report of 50 patients with $\mathrm{S}$. aureus catheter-related sepsis, delayed catheter removal was associated with increased mortality [53]. Similarly, in a meta-analysis of 11 studies, 8 of 132 patients who had a short course of treatment for S. aureus developed endocarditis or infection at another site [54]. Failure or delay in removing the catheter increases the risk for haematogenous complications $[49,55]$. In the largest published series on the use of antibiotic lock for CRBSI due to S. aureus, treatment failure was observed in one-half of cases [52]. This needs to be taken into account when attempting catheter salvage.

Echocardiography should be carried out in at-risk patients to diagnose possible endocarditis, which requires therapy for 46 weeks $[56,57]$. Patients who are being considered for a shorter duration of therapy should also have transoesophageal echocardiography, which is most sensitive when performed 57 days after the onset of bacteraemia [58].

The duration of therapy should be based on the likelihood of deepseated complications. A longer course of therapy is prudent for immunosuppressed patients with S. aureus CRBSI. If there is evidence of other distant foci of infection, such as septic venous thrombosis, treatment should similarly be continued for several weeks. Patients whose catheter has been removed and have a negative echocardiogram should be treated with appropriate systemic antibiotics for $10-14$ days $[56,57]$. 
The types of antibiotic used should be based on the susceptibility of

S. aureus. For meticillin-sensitive S. aureus a semisynthetic antistaphylococcal penicillin or first-generation cephalosporin is the first choice [39]. For MRSA, several options can be considered, including vancomycin, daptomycin or even linezolid.

\subsection{Enterococcus spp.}

The proportion of enterococcal bacteraemias associated with CVCs has increased dramatically since the early 1980s [4-6], reaching $35 \%$ in one UK study [59]. Management of enterococcal CRBSI is an increasing clinical challenge for which specific clinical data are lacking. Like many other organisms capable of causing CRBSI, enterococci can adhere to biomedical polymers and form a biofilm [60], thereby making antimicrobial treatment problematic.

Secondary endocarditis may complicate the management of enterococcal CRBSI [61,62]. Unlike S. aureus CRBSI [34] secondary enterococcal endocarditis appears to be a rare complication, but if the patient has signs and symptoms of endocarditis, persistent bacteraemia or enterococcal bacteraemia in the presence of a prosthetic valve a transoesophageal echocardiograph should be done $[59,63]$. The IDSA guidelines for the treatment of enterococcal CRBSI caused by susceptible isolates advise either ampicillin or vancomycin alone or in combination with an aminoglycoside [39], as data concerning the role of combination therapy for treatment 
are conflicting. In cases of CRBSI due to ampicillin- and vancomycin-resistant enterococci, linezolid or daptomycin may be used based on antibiotic susceptibility results.

If a long-term catheter is retained in cases of uncomplicated infection 7-14 days of intravenous treatment is recommended in addition to antibiotic lock therapy [64].

\subsection{Gram-negative bacilli}

Over the past decade, although the rates of Gram-negative bacillary infection of intravascular devices in adults have decreased, there has been great concern over the increasing incidence of Gramnegative bacilli resistant to next-generation cephalosporins and the evolution of multidrug-resistant Gram-negative bacilli that produce carbapenemases $[2,65]$.

In general, data concerning the management of CRBSI due to Gram-negative bacilli are limited. Hanna and colleagues [66] showed that documented CRBSIs caused by Gram-negative bacilli were associated with a high frequency of relapse if the CVC was retained. Similarly, with P. aeruginosa CVC sepsis catheter removal also improved patient outcome [67]. Failure to remove the catheter was associated with a significantly higher rate of treatment failure and bacteraemia recurrence $(P<0001)$. Several studies suggest that if the Gram-negative bacillary bacteraemia is judged to be a CRBSI, especially with bacilli that have a propensity for biofilm 
production, such as Acinetobacter baumannii, Pseudomonas spp. and Stenotrophomonas maltophilia [68-70], then it is prudent to remove the CVC [39].

CRBSI caused by S. maltophilia is still rare and occurs mostly in immunocompromised hosts. Catheter removal seems to be crucial, since $92 \%$ of patients with catheter removal survived, compared with only $54 \%$ without catheter removal [69].

Results from recent studies in which Gram-negative CRBSI were treated with antibiotic lock therapy with compounds with activity against Gram-negative bacteria, such as gentamicin, amikacin or ceftazidime, and systemic antibiotics, have been encouraging $[52,71]$. Septic, critically ill or neutropenic patients and patients having a femoral catheter or a known focus of infection are at higher risk for infection due to multidrug-resistant Gram-negative bacilli and should receive empirical antibiotic therapy according to recent IDSA guidelines. Critically ill patients with suspected CRBSI and who have recent colonization or infection with a multidrugresistant Gram-negative pathogen should receive initial therapy with two antimicrobial agents of different classes with Gramnegative activity while waiting for culture and susceptibility results. Finally, evaluation for endovascular infection and metastatic infection is necessary in cases of persistent bacteraemia or severe sepsis despite systemic and antibiotic lock therapy, and intravenous 
antibiotic treatment is needed beyond 7-14 days after the removal of the device.

\subsection{Candida spp.}

The presence of a vascular catheter or other medical device increases a patient's risk for invasive candidiasis by a factor of seven. Candida spp. are the second most common cause of these device-related infections and are associated with the highest pathogen-specific mortality [72]. The burden of this infection has risen more than $200 \%$ in the past two decades and is associated with high mortality ( $>40 \%$ ), extended hospital stays and high cost [73].

Many prospective studies show that CVC retention worsened outcome; CVC retention for $>72 \mathrm{~h}$ was associated with a poorer outcome in a study of 404 patients with candidaemia and indwelling CVC $[39,40]$. The CVC should therefore be removed within $72 \mathrm{~h}$ in patients with suspected or documented catheter-related candidaemia, and the catheter tip should be sent for culture. In cases of limited venous access or severe thrombocytopenia in patients with candidaemia and a short-term CVC, for whom no source of candidaemia is obvious, the catheter could be exchanged over a guidewire and sent for culture, and removed if it is colonized with the same species of Candida as found in a percutaneous blood culture. 
In patients with catheter-related candidaemia, fluconazole or an echinocandin should be considered as an efficacious and safer alternative to amphotericin $B$, although in centres where there are Candida spp. with decreased susceptibility to azoles (e.g. C. glabrata and C. krusei) an echinocandin should be used. According to IDSA guidelines antifungal therapy is recommended for all cases of CRBSI due to Candida spp., including cases in which clinical manifestations of infection and/or candidaemia resolve after catheter withdrawal and before initiation of antifungal therapy. The duration of therapy for uncomplicated catheter-related candidaemia should be 2 weeks after the last positive blood culture.

There are limited data on the efficacy of antifungal lock solutions. Compared with bacterial infection, Candida CRBSI is more difficult to eradicate with antibiotic lock therapy [74-76]. Echinocandins, lipid formulations of amphotericin B and ethanol-based lock solutions have been studied, and seem to be effective in vitro, but further clinical studies are required in the setting of catheter-related candidaemia.

\subsection{Other microorganisms}

Infections due to other skin microflora, such as Corynebacterium and Bacillus spp. are infrequent causes of CRBSI and the treatment approach is quite similar to that for S. epidermidis. Confirmation by multiple percutaneous blood culture results positive for the same 
organism is required before meaningful conclusions can be drawn as to the significance of the culture results [77].

Catheter removal is usually indicated for the management of these infections unless there are no alternative intravascular access sites. CVC removal might not be necessary in patients with corynebacterial CRBSI, particularly if systemic therapy consists of non-glycopeptide antibiotics [78].

Bacillus spp. are common contaminants of blood cultures but clinically significant infection is rare $[79,80]$. Significant CVC infections caused by Bacillus spp. are mainly due to B. cereus and have mostly been reported in immunocompromised hosts [81].CVC infection with Bacillus spp. usually requires catheter removal for complete cure $[82,83]$. In a patient with AIDS, B. subtilis bacteraemia resolved with vancomycin therapy without removing the Broviac catheter.

Micrococcus spp. are a common cause of BSI in patients with pulmonary hypertension on intravenous epoprostenol, but are rarely reported in other groups of patients and their presence on a blood culture should not be interpreted as skin contamination [84]. The clinical presentation can be acute or subacute. Treatment should be based on the organism's antibiotic susceptibilities and removal of the line should be considered if the symptoms or the bacteraemia persists. 
Tsukamurella spp. (aerobic Gram-positive organisms from the order Actinomycetales) are infrequent causes of CRBSI. In a recent review, Bouza and colleagues noted that 12 of 14 patients with CRBSI caused by Tsukamurella spp. were being treated for an underlying malignancy, while usual underlying morbidities include primary immunodeficiencies, a variety of malignancies and chronic renal failure [85]. In the setting of CRBSI caused by a Tsukamurella sp., treatment in all reported cases ultimately required the removal of the infected catheter in addition to antimicrobial therapy $[86,87]$. The literature provides no firm conclusions regarding the ideal choice or duration of antimicrobial therapy for this infection.

Rapidly growing non-tuberculous mycobacteria are responsible for a variety of clinical syndromes in humans, including CRBSIs. Mycobacteria should be included in the differential diagnosis of CVCrelated sepsis, particularly for immunocompromised hosts [88]. M. fortuitum complex (M. fortuitum and M. chelonae) is a cause of catheter-related bacteraemia in patients with cancer. Appropriate treatment consists of antibiotic therapy and catheter removal [89]. Tunnel infections usually also require surgical excision.

CRBSIs due to Achromobacter xylosoxidans, a Gram-negative rod, have been observed in patients with cancer or AIDS $[90,91]$. Despite the bacterium's resistance to aminoglycosides the patients were treated successfully with a combination of ceftazidime and amikacin without removing the catheter. Infections occurring in 
neutropenic patients were successfully treated with imipenem and catheter removal [92] and in the first reported outbreak of CVCrelated bacteraemia due to $A$. xylosoxidans, patients were successfully treated with intravenous levofloxacin and antibiotic lock without catheter removal [93].

Agrobacterium spp. are saprophytic bacilli and opportunistic pathogens of humans, only 25 cases of infection being described by 1997. The biofilm-forming species Rhizobium (Agrobacterium) radiobacter can cause community catheter-related infections. Most cases respond to treatment only after removal of the catheter and antimicrobial susceptibility seems to be variable [94].

\section{Conclusions}

CRBSI is an iatrogenic problem that causes significant morbidity and mortality, excess length of stay and excess costs. The risk of infection is highest with percutaneous CVCs, somewhat lower with tunnelled or subcutaneous catheters and lowest with peripheral intravenous catheters. Management of CRBSI involves deciding on catheter removal, antimicrobial lock solution and the type and duration of systemic antimicrobial therapy. A distinction should be made between complicated and uncomplicated CRBSI. The identity of the organism is an important factor to be considered. Once infection occurs the line should generally be removed, unless there are major contraindications. Long-term catheters should be 
removed in patients with infections due to S. aureus, P. aeruginosa, fungi or mycobacteria. Short-term catheters should be removed in patients with CRBSI due to Gram-negative bacilli, S. aureus, enterococci, fungi or mycobacteria. The antibiotic lock technique is promising and easy to perform and may help catheter retention in certain situations.

Antimicrobial therapy is initially empirical and should be directed against suspected microorganisms (usually staphylococci) and modified on receipt of the results of cultures.

Funding: The author received an honorarium for writing this article. The funds for the honorarium were provided by Novartis AG, Switzerland and were handled by the organizing committee of the 4th European Conference on Bloodstream Infections for the publication of this supplement.

Competing interests: No competing interests.

Ethical approval: Approved by the ethical committee of Patras University Hospital. 
References

1. Mermel LA. Prevention of intravascular catheter-related infections. Ann Intern Med 2000;132:391-402.

2. CDC. National Nosocomial Infections Surveillance (NNIS) System report, data summary from October 1986-April 1998, issued June1998. Am J Infect Control 1998;26:522-33.

3. Collignon PJ. Intravascular catheter-associated sepsis: a common problem. The Australian Study on Intravascular Catheter Associated Sepsis. Med J Aust 1994;161:374-8.

4. Heiselman D. Nosocomial bloodstream infections in the critically ill. JAMA $1994 ; 272: 1819-20$.

5. Dimick JB, Pelz RK, Consunji R, Swoboda SM, Hendrix CW, Lipsett PA. Increased resource use associated with catheter-related bloodstream infection in the surgical intensive care unit. Arch Surg $2001 ; 136: 229-34$

6. Kluger DM, Maki DG. The relative risk of intravascular devicerelated bloodstream infections in adults. In: Program and abstracts of the 39th Interscience Conference on Antimicrobial Agents and Chemotherapy; 26-29 September 1999; San Francisco, CA. Washinton, DC: American Society for Microbiology;1999, Abs 1913. 7. O'Grady NP, Alexander M, Dellinger EP, Gerberding JL, Heard SO, Maki DG et al; Healthcare Infection Control Practices Advisory 
committee. Guidelines for the prevention of intravascular catheterrelated infections. Infect Control Hosp Epidemiol 2002;23:759-69.

8. Managing bloodstream infections associated with intravascular catheters. Drug Ther Bull 2001;39:75-80.

9. Maki DG, Kluger DM, Crnich CJ. The risk of bloodstream infection in adults with different intravascular devices: a systematic review of 200 published prospective studies. Mayo Clin Proc $2006 ; 81: 1159-71$.

10. McGee DC, Gould MK. Preventing complications of central venous catheterization. N Engl J Med 2003;348:1123-33.

11. Horvath R, Collignon P. Controlling intravascular catheter infections. Aust Prescr 2003;26:41-3.

12. Sitges-Serra A, Linares J, Perez JL, Jaurrieta E, Lorente L. A randomized trial on the effect of tubing changes on hub contamination and catheter sepsis during parenteral nutrition. JPEN J Parenter Enteral Nutr 1985;9:322-5.

13. Mermel LA, McCormick RD, Springman SR, Maki DG. The pathogenesis and epidemiology of catheter-related infection with pulmonary artery Swan-Ganz catheters: a prospective study utilizing molecular subtyping. Am J Med 1991;91(Suppl 3B):S197205. 
14. Bouza E, Burillo A, Munoz P. Catheter-related infections: diagnosis and intravascular treatment. Clin Microbiol Infect $2002 ; 8: 265-74$.

15. Raad I. Intravascular catheter-related infections. Lancet $1998 ; 351: 893-8$.

16. Donlan RM. Biofilms and device-associated infections. Emerg Infect Dis $2001 ; 7: 277-81$.

17. Balaban N, Gov Y, Bitler A, Boelaert JR. Prevention of Staphylococcus aureus biofilm on dialysis catheters and adherence to human cells. Kidney Int $2003 ; 63: 340-5$.

18. Lowy FD. Staphylococcus aureus infections. N Engl J Med $1998 ; 339: 520-32$.

19. Balaban N, Goldkorn T, Gov Y, Hirshberg M, Koyfman N, Matthews HR, et al: Regulation of Staphylococcus aureus pathogenesis via target of RNAIII-activating protein (TRAP). J Biol Chem 2001;276:2658-67.

20. Ludwicka A, Uhlenbruck G, Peters G, Seng PN, Gray ED, Jeljaszewicz J, et al. Investigation on extracellular slime substance produced by Staphylococcus epidermidis. Zentralbl Bakteriol Mikrobiol Hyg 1984;258:256-67.

21. Ramage G, Saville SP, Thomas DP, López-Ribot JL. Candida biofilms: an update. Eukaryot Cell 2005;4:633-8. 
22. Douglas LJ. Candida biofilms and their role in infection. Trends Microbiol 2003;11:30-6.

23. Kuhn DM, Chandra J, Mukherjee PK, Ghannoum MA.

Comparison of biofilms formed by Candida albicans and Candida parapsilosis on bioprosthetic surfaces. Infect Immun 2002;70:87888.

24. Kumamoto CA. Candida biofilms. Curr Opin Microbiol $2002 ; 5: 608-11$.

25. García-Sánchez S, Aubert S, Iraqui I, Janbon G, Ghigo JM, d'Enfert C. Candida albicans biofilms: a developmental state associated with specific and stable gene expression patterns. Eukaryot Cell 2004;3:536-45.

26. Bachmann SP, Ramage G, VandeWalle K, Patterson TF, Wickes BL, López-Ribot JL. Antifungal combinations against Candida albicans biofilms in vitro. Antimicrob Agents Chemother $2003 ; 47: 3657-9$.

27. Hogan DA, Kolter R. Pseudomonas-Candida interactions: an ecological role for virulence factors. Science $2002 ; 296: 2229-32$.

28. Hogan DA, Vik A, Kolter R. A Pseudomonas aeruginosa quorum-sensing molecule influences Candida albicans morphology. Mol Microbiol 2004;54:1212-23. 
29. Bouza E, San Juan R, Munoz P, Pascau J, Voss A, Desco M. A European perspective on intravascular catheter-related infections: report on the microbiology workload, aetiology and antimicrobial susceptibility (ESGNI-005 Study). Clin Microbiol Infect $2004 ; 10: 838-42$.

30. Linde HJ, Hahn J, Holler E, Reischl U, Lehn N. Septicemia due to Acinetobacter junii. J Clin Microbiol 2002;40:2696-7.

31. Crump JA, Collignon PJ. Intravascular catheter-associated infections. Eur J Clin Microbiol Infect Dis 2000;19:1-8.

32. Schaberg DR, Culver DH, Gaynes RP. Major trends in the microbial etiology of nosocomial infection. Am J Med1991;91(Suppl):S72-75.

33. Amann R, Ludwig W, Schleifer KH. Phylogenetic identification and in situ detection of individual microbial cells without cultivation. Microbiol Rev 1995;59:143-69.

34. Tumbarello M, Spanu T, Sanguinetti M, Citton R, Montuori E, Leone $\mathrm{F}$, et al. Bloodstream infections caused by extendedspectrum-ß-lactamase-producing Klebsiella pneumoniae: Risk factors, molecular epidemiology, and clinical outcome. Antimicrob Agents Chemother 2006;50:498-504.

35. Hidron AI, Edwards JR, Patel J, Horan TC, Sievert DM, Pollock D, et al for the NHSN Team and Participating Facilities. NHSN annual update: antimicrobial resistant pathogens associated with 
healthcare-acquired infections (HAIs) reported to the National Healthcare Safety Network at the Centers for Disease Control and Prevention, 2006-7. Infect Control Hosp Epidemiol 2008;29:9961011. Erratum in: Infect Control Hosp Epidemiol 2009;30:107.

36. Larsen MK, Thomsen TR, Moser C, Høiby N, Nielsen PH. Use of cultivation-dependent and -independent techniques to assess contamination of central venous catheters: a pilot study. BMC Clin Pathol 2008;8:10.

37. Chatzinikolaou I, Hanna H, Hachem R, Alakech B, Tarrand J, Raad I. Differential quantitative blood cultures for the diagnosis of catheter-related bloodstream infections associated with short- and long-term catheters: a prospective study. Diagn Microbiol Infect Dis $2004 ; 50: 167-2$.

38. Maki D, Weise C, Sarafin H. A semiquantitative culture method for identifying intravenous catheter-related infection. N Engl J Med 1977;296:1305-9.

39. Mermel LA, Allon M, Bouza E, Craven DE, Flynn P, O'Grady NP, et al. Clinical practice guidelines for the diagnosis and management of intravascular catheter-related infection: 2009 update by the Infectious Diseases Society of America. Clin Infect Dis $2009 ; 49: 1-45$.

40. Capdevila JA, Segarra A, Planes AM, Ramírez-Arellano M, Pahissa A, Piera L, et al. Successful treatment of haemodialysis 
catheter-related sepsis without catheter removal. Nephrol Dial Transplant 1993;8:231-4.

41. Messing B, Peitra-Cohen S, Debure A, Beliah M, Bernier J. Antibiotic-lock technique: a new approach to optimal therapy for catheter-related sepsis in home-parenteral nutrition patients. JPEN J Parenter Enteral Nutr 1988;12:185-9.

42. Raad I. Management of intravascular catheter-related infections. Antimicrob Chemother 2000;45:267-70.

43. Anthony TU, Rubin LG. Stability of antibiotics used for antibiotic lock treatment of infections of implantable venous devices (ports). Antimicrob Agents Chemother 1999;43:2074-6.

44. FDA. FDA information for healthcare professionals: Linezolid (marketed as Zyvox). FDA alert: March 16, 2007.

http://www.fda.gov/Drugs/DrugSafety/PostmarketDrugSafetyInformationf orPatientsandProviders/ucm101503.htm [accessed 8 October 2010].

45. Wilcox MH, Tack KJ, Bouza E, Herr DL, Ruf BR, Ijzerman MM, et al. Complicated skin and skin-structure infections and catheterrelated bloodstream infections: noninferiority of linezolid in a phase 3 study. Clin Infect Dis 2009;48:203-12.

46. Zinkernagel AS, Zinkernagel MS, Elzi MV, Genoni M, Gubler J, Zbinden R, et al. Significance of Staphylococcus lugdunensis bacteremia: report of 28 cases and review of the literature. Infection $2008 ; 36: 314-2$. 
47. Lederer SR, Riedelsdorf G, Schiffl H. Nasal carriage of meticillin-resistant Staphylococcus aureus: the prevalence, patients at risk and the effect of elimination on outcomes among outclinic haemodialysis patients. Eur J Med Res 2007;12:284-8.

48. Raad I, Hanna H, Maki D. Intravascular catheter-related infections: advances in diagnosis, prevention, and management. Lancet Infect Dis 2007;7:645-57.

49. Fowler VG Jr, Justice A, Moore C, Benjamin DK Jr, Woods CW, Campbell S, et al. Risk factors for hematogenous complications of intravascular catheter-associated Staphylococcus aureus bacteremia. Clin Infect Dis 2005;40:695-703.

50. Fowler VG Jr, Miro JM, Hoen B, Cabell CH, Abrutyn E, Rubinstein E, et al. Staphylococcus aureus endocarditis: a consequence of medical progress. JAMA 2005;293:3012-21.

51. Rijnders BJ, Van Wijngaerden E, Vandecasteele SJ, Stas M, Peetermans WE. Treatment of long-term intravascular catheterrelated bacteremia with antibiotic lock: randomized, placebocontrolled trial. J Antimicrob Chemother 2005;55:90-4.

52. Fernandez-Hidalgo N, Almirante B, Calleja R, Ruiz I, Planes AM, Rodriguez D, et al. Antibiotic-lock therapy for long-term intravascular catheter-related bacteraemia: results of an open, noncomparative study. J Antimicrob Chemother 2006;57:1172-80. 
53. Malanoski GJ, Samore MH, Pefanis A, Karchmer AW. Staphylococcus aureus catheter-associated bacteremia. Minimal effective therapy and unusual infectious complications associated with arterial sheath catheters. Arch Intern Med 1995;155:1161-6.

54. Jernigan JA, Farr BM. Short-course therapy of catheter-related Staphylococcus aureus bacteremia: a meta-analysis. Ann Intern Med 1993;119:304-11.

55. Fowler VG Jr, Sanders LL, Sexton DJ, Kong L, Marr KA, Gopal AK, et al. Outcome of Staphylococcus aureus bacteremia according to compliance with recommendations of infectious diseases specialists: experience with 244 patients. Clin Infect Dis $1998 ; 27: 478-86$.

56. Benoit JL, Carandang G, Sitrin M, Arnow PM. Intraluminal antibiotic treatment of central venous catheter infections in patients receiving parenteral nutrition at home. Clin Infect Dis $1995 ; 21: 1286-8$.

57. Johnson DC, Johnson FL, Goldman S. Preliminary results treating persistent central venous catheter infections with the antibiotic lock technique in pediatric patients. Pediatr Infect Dis J $1994 ; 13: 930-93$.

58. Sochowski RA, Chan KL. Implication of negative results on a monoplane transesophageal echocardiographic study in patients 
with suspected infective endocarditis. J Am Coll Cardiol $1993 ; 21: 216-21$.

59. Sandoe JA, Witherden IR, Au-Yeung HK, Kite P, Kerr KG, Wilcox MH. Enterococcal intravascular catheter-related bloodstream infection: management and outcome of 61 consecutive cases. J Antimicrob Chemother 2002;50:577-82.

60. Joyanes P, Pascual A, Martínez-Martínez L, Hevia A, Perea EJ. In vitro adherence of Enterococcus faecalis and Enterococcus faecium to plastic biomaterials. Clin Microbiol Infect 1999;5:382-6.

61. Venditti M, Biavasco F, Varaldo PE, Macchiarelli A, De Biase L, Marino B, et al. Catheter-related endocarditis due to glycopeptideresistant Enterococcus faecalis in a transplanted heart. Clin Infect Dis $1993 ; 17: 524-5$.

62. Fernández-Guerrero ML, Verdejo C, Azofra J, de Górgolas M. Hospital-acquired infectious endocarditis not associated with cardiac surgery: An emerging problem. Clin Infect Dis 1995;20:16-23.

63. Fernandez-Guerrero ML, Herrero L, Bellver M, Gadea I, Roblas RF, de Gorgolas M. Nosocomial enterococcal endocarditis: a serious hazard for hospitalized patients with enterococcal bacteraemia. J Intern Med 2002;252:510-5.

64. Fortún J, Grill F, Martín-Dávila P, Blázquez J, Tato M, Sánchez-Corral J, et al. Treatment of long-term intravascular 
catheter-related bacteraemia with antibiotic-lock therapy. J

Antimicrob Chemother 2006;58:816-21.

65. Jacoby GA, Munoz-Price LS. The new $\beta$-lactamases. $N$ Engl J Med 2005;352:380-91.

66. Hanna H, Afif C, Alakech B, Boktour M, Tarrand J, Hachem R, et al. Central venous catheter-related bacteraemia due to Gramnegative bacilli: significance of catheter removal in preventing relapse. Infect Control Hosp Epidemiol 2004;25: 646-49.

67. Kuikka A, Valtonen VV. Factors associated with improved outcome of Pseudomonas aeruginosa bacteraemia in a Finnish university hospital. Eur J Clin Microbiol Infect Dis 1998;17:701-8.

68. Seifert H, Strate A, Pulverer G. Nosocomial bacteremia due to Acinetobacter baumannii. Clinical features, epidemiology, and predictors of mortality. Medicine (Baltimore) 1995;74:340-9.

69. Friedman ND, Korman TM, Fairley CK, Franklin JC, Spelman DW. Bacteraemia due to Stenotrophomonas maltophilia: an analysis of 45 episodes. J Infect 2002;45:47-53.

70. Seifert H. Catheter-related infections due to Gram-negative bacilli. In: Seifert $H$, Jansen B, Farr BM, editors. Catheter-related infections. New York: Marcel Dekker; 1997, p. 111. 
71. Poole CV, Carlton D, Bimbo L, Allon M. Treatment of catheterrelated bacteraemia with an antibiotic lock protocol: effect of bacterial pathogen. Nephrol Dial Transplant 2004;19:1237-44.

72. Kojic EM, Darouiche RO. Candida infections of medical devices. Microbiol Rev 2004;17: 255-67.

73. Wenzel RP. Nosocomial candidemia: risk factors and attributable mortality. Clin Infect Dis 1995;20:1531-4.

74. Krzywda EA, Andris DA, Edmiston CE Jr, Quebbeman EJ. Treatment of Hickman catheter sepsis using antibiotic lock technique. Infect Control Hosp Epidemiol 1995;16:596-8.

75. Benoit JL, Carandang G, Sitrin M, Arnow PM. Intraluminal antibiotic treatment of central venous catheter infections in patients receiving parenteral nutrition at home. Clin Infect Dis $1995 ; 21: 1286-8$.

76. Arnow PM, Kushner R. Malassezia furfur catheter infection cured with antibiotic lock therapy. Am J Med 1991;90:128-30.

77. Hall KK, Lyman JA. Updated review of blood culture contamination. Clin Microbiol Rev 2006;19:788-802.

78. Ghide S, Jiang Y, Hachem R, Chaftari AM, Raad I. Catheterrelated Corynebacterium bacteremia: should the catheter be removed and vancomycin administered? Eur J Clin Microbiol Infect Dis $2010 ; 29: 153-6$. 
79. Weber DJ, Saviteer SM, Rutala WA, Thomann CA. Clinical significance of Bacillus species isolated from blood cultures. South Med J 1989;82:705-9.

80. Pearson HE: Human infections caused by organisms of the Bacillus genus. Am J Clin Pathol 1970;53:506-15.

81. Drobniewski FA. Bacillus cereus and related species. Clin Microbiol Rev 1993;6:324-38.

82. Salzman MB, Rubin LG. Intravenous catheter-related infections. Adv Pediatr Infect Dis 1995;10:337-68.

83. Sitges-Serra A, Girvent M. Catheter-related bloodstream infections. World J Surg 1999;23:589-95.

84. Oudiz RJ, Widlitz A, Beckmann XJ, Camanga D, Alfie J, Brundage $\mathrm{BH}$, et al. Micrococcus-associated central venous catheter infection in patients with pulmonary arterial hypertension. Chest $2004 ; 126: 90-4$.

85. Bouza E, Pérez-Parra A, Rosal M, Martín-Rabadán P, Rodríguez-Créixems M, Marín M. Tsukamurella: a cause of catheterrelated bloodstream infections. Eur J Clin Microbiol Infect Dis $2009 ; 28: 203-10$.

86. Jones RS, Fekete T, Truant AL, Satishchandran V. Persistent bacteremia due to Tsukamurella paurometabolum in a patient 
undergoing hemodialysis: case report and review. Clin Infect Dis $1994 ; 18: 830-2$.

87. Elshibly S, Doherty J, Xu J, McClurg RB, Rooney PJ, Millar BC, et al. Central line-related bacteraemia due to Tsukamurella tyrosinosolvens in a haematology patient. Ulster Med J 2005;74:436. 88. Hawkins C, Qi C, Warren J, Stosor V. Catheter-related bloodstream infections caused by rapidly growing nontuberculous mycobacteria: a case series including rare species. Diagn Microbiol Infect Dis 2008;61:187-91.

89. Raad II, Vartivarian S, Khan A, Bodey GP. Catheter-related infections caused by the Mycobacterium fortuitum complex: 15 cases and review. Rev Infect Dis 1991;13:1120-5.

90. Legrand C, Anaissie E. Bacteremia due to Achromobacter xylosoxidans in patients with cancer. Clin Infect Dis $1992 ; 14: 479-$ 84.

91. Manfredi R, Nanetti A, Ferri M, Chiodo F. Bacteremia and respiratory involvement by Alcaligenes xylosoxidans in patients infected with the human immunodeficiency virus. Eur J Clin Microbiol Infect Dis 1997;16:933-8.

92. Hernández JA, Martino R, Pericas R, Sureda A, Brunet S, Domingo-Albós A. Achromobacter xylosoxidans bacteremia in 
patients with hematologic malignancies. Haematologica $1998 ; 83: 284-86$.

93. Tena D, Carranza R, Barberá JR, Valdezate S, Garrancho JM, Arranz M, et al. Outbreak of long-term intravascular catheterrelated bacteremia due to Achromobacter xylosoxidans subspecies xylosoxidans in a hemodialysis unit. Eur J Clin Microbiol Infect Dis $2005 ; 24: 727-32$.

94. Hulse M, Johnson S, Ferrieri P. Agrobacterium infections in humans: experience at one hospital and review. Clin Infect Dis $1993 ; 16: 112-7$. 